\title{
Pancreatic Giant Cell Tumor: A Variant of Pancreatic Adenocarcinoma with Aggressive Course
}

\author{
Suhas Kodasoge Rajappa ${ }^{3}$ Amrith BP² \\ ${ }^{1}$ Department of Radiology, Rajiv Gandhi Cancer Institute and \\ Research Centre, New Delhi, India \\ ${ }^{2}$ Department of Medical Oncology, Rajiv Gandhi Cancer Institute \\ and Research Centre, New Delhi, India \\ ${ }^{3}$ Department of Surgical Oncology, Rajiv Gandhi Cancer Institute \\ and Research Centre, New Delhi, India
}

Ankush Jajodia ${ }^{1}$ Venkata Pradeep Babu Koyyala ${ }^{2}$ Varun Goel ${ }^{2}$ Prasantha Kumar Dash ${ }^{2}$

\begin{abstract}
Address for correspondence Venkata Pradeep Babu Koyyala, MD, Room: 2262, 2nd Floor, C Block, Rajiv Gandhi Cancer Institute and Research Centre, Sector 5, Rohini,New Delhi 110085, India (e-mail: pradeepbabu.koyyala@gmail.com).
\end{abstract}

Asian J Oncol 2018;4:79-80

\begin{abstract}
Ductal adenocarcinoma of pancreas is the most common pancreatic neoplasm and is the third leading cause of death due to cancer, with a 5-year survival of $8 \%$ only. Although

Keywords

- osteoclastic

- pleomorphic

- giant cell tumor

- pancreas pancreatic neoplasms are broadly classified into endocrine and exocrine variety based on cell of origin, on imaging they are classified into solid and cystic types. Pancreatic giant cell tumor (PGCT) is a very uncommon and aggressive pancreatic neoplasm. We report a case of PGCT presenting as a pancreatic tail mass and liver lesions with a morphological overlap with other cystic benign and malignant lesions of pancreas on imaging.
\end{abstract}

\section{Introduction}

Ductal adenocarcinoma of pancreas is the most common pancreatic neoplasm and is the third leading cause of death due to cancer, with a 5 -year survival of $8 \%$ only. Although pancreatic neoplasms are broadly classified into endocrine and exocrine variety based on cell of origin, on imaging they are classified into solid and cystic types. Pancreatic giant cell tumor (PGCT) is a very uncommon and aggressive pancreatic neoplasm. We report a case of PGCT presenting as a pancreatic tail mass and liver lesions with a morphological overlap with other cystic benign and malignant lesions of pancreas on imaging.

\section{Case Report}

A 47-year-old woman who was housewife presented with epigastric pain, vomiting, and weight loss for past 4 months. There was no prior history of alcohol intake or smoking. Abdominal examination revealed an ill-defined mass in the epigastric region with mild tenderness. Laboratory results revealed normal amylase and lipase levels and elevated levels of carbohydrate antigen (CA) 19-9 serum marker and serum alkaline phosphatase. Diagnostic positron emission tomography-computed tomography (PET-CT) revealed an ill-defined metabolically active large lobulated solid/cystic soft tissue mass $(11.1 \times 9.8 \times 16.0 \mathrm{~cm}$, standardized uptake value [SUV] max 6.9) seen to arise from pancreatic tail region ( - Fig. 1). Metabolically active splenic venous thrombosis was seen (SUV max 9.3) reaching till confluence and extending into adjacent portal vein. Liver also revealed multiple metabolically active hypodense lesions in both lobes (largest in segment VIII $3.5 \times 3.1 \mathrm{~cm}$, SUV max 7.7) (-Fig. 2). CT-guided biopsy yielded multiple fragmented soft tissue cores measuring 0.6 to $0.8 \mathrm{~cm}$. Histopathologic examination (HPE) revealed infiltration by diffuse sheets of tumor cells having moderate eosinophilic to fine granular and vacuolated cytoplasm with indistinct borders, round vesicular nucleus, and prominent nucleoli. Fine fibrovascular septa were seen separating the tumor cells with occasional mitosis and

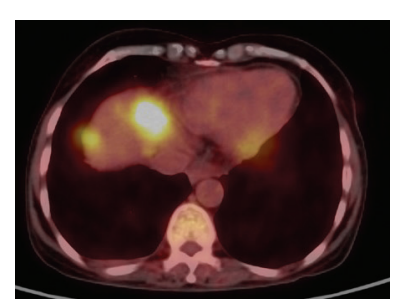

Fig. 1 Metabolically active splenic venous thrombosis was seen (SUV max 9.3) reaching till confluence and extending into adjacent portal vein.
DOI https://doi.org/ 10.1055/s-0039-1680841 ISSN 2454-6798.
License terms

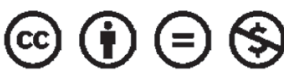




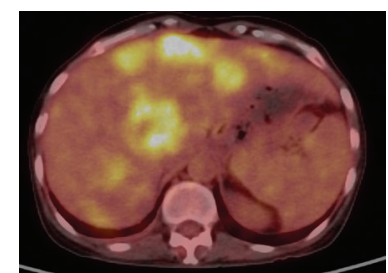

Fig. 2 PET-CT showing multiple metabolically active hypodense lesions in both lobes of liver (largest in segment VIII $3.5 \times 3.1 \mathrm{~cm}$, SUV max 7.7). PET-CT, positron emission tomography-computed tomography.

areas of necrosis. Numerous multinucleated giant cells were seen. Overall the impression was that of a poorly differentiated neoplasm. Further immunohistochemistry characterization showed that tumor cells expressed CD68, CD163, and focal CK. These were negative for PAX-8, CEA, CK-7, CK-20, and synaptophysin, hence labeled as pleomorphic PGCT.

In this patient, we were unable to start treatment due to very poor performance status of the patient. The patient succumbed within 15 days of presentation. This atypical case highlights the challenges in diagnosing pancreatic lesions and emphasizes the significance of considering less common forms of pancreatic tail masses and poor prognosis of variants of pancreatic carcinoma.

PGCTs are unusual nonendocrine tumors of the pancreas with prevalence less than $1 \%$ of all pancreatic tumors, first described by Sommers and Meissner in 1954. ${ }^{1}$ Three types of PGCT have been described based on histology: osteoclastic, pleomorphic, and mixed. ${ }^{2}$ Later in 2010, the World Health Organization (WHO) grouped them together as undifferentiated carcinoma with osteoclast-like giant cells. ${ }^{3}$
Clinical manifestations of pleomorphic giant cell carcinoma are analogous to those of pancreatic adenocarcinoma with abdominal pain and weight loss being the most prevalent. ${ }^{4}$ The survival range for pleomorphic giant cell pancreatic cancer ranges from several weeks in advanced unresectable disease to 25 months. Osteoclastic giant cell tumors may behave less aggressively than other PGCT. ${ }^{5}$ The lack of evidence upon which to base treatment decisions leads to difficulty in formative assessment of multidisciplinary approach for each patient.

\section{Conflict of Interest}

None declared.

\section{References}

1 Burkadze G, Turashvili G. A case of osteoclast-like giant cell tumor of the pancreas associated with borderline mucinous cystic neoplasm. Pathol Oncol Res 2009;15(1):129-131

2 Athanasios P, Alexandros P, Nicholas B, et al. Pleomorphic giant cell carcinoma of the pancreas with hepatic metastases-initially presenting as a benign serous cystadenoma: a case report and review of the literature. HPB Surg 2010;2010:627360

3 Lukás Z, Dvorák K, Kroupová I, Valásková I, Habanec B. Immunohistochemical and genetic analysis of osteoclastic giant cell tumor of the pancreas. Pancreas 2006;32(3):325-329

4 Tschang TP, Garza-Garza R, Kissane JM. Pleomorphic carcinoma of the pancreas: an analysis of 15 cases. Cancer 1977;39(5):2114-2126

5 Lewandrowski KB, Weston L, Dickersin GR, Rattner DW, Compton CC. Giant cell tumor of the pancreas of mixed osteoclastic and pleomorphic cell type: evidence for a histogenetic relationship and mesenchymal differentiation. Hum Pathol 1990;21(11):1184-1187 